\title{
Radially Distributed Values of Holomorphic Curves
}

\section{Nan Wu ${ }^{1}$}

Received: 25 September 2016 / Revised: 14 November 2016 / Accepted: 3 January 2017 /

Published online: 26 June 2017

(C) The Author(s) 2017. This article is an open access publication

Abstract Using the spread relation we investigate the growth of transcendental holomorphic curves when they have radially distributed small holomorphic curves.

Keywords Holomorphic curves $\cdot$ Spread relation · Radial distribution

Mathematics Subject Classification Primary 30D10; Secondary 30D20 - 30B10 . 34M05

\section{Introduction}

Let $f(z)$ be a transcendental meromorphic function on the complex plane $\mathbb{C}$ and let $a(z)$ be a meromorphic function satisfying

$$
T(r, a)=o(T(r, f)), \quad r \rightarrow \infty .
$$

Then $a(z)$ is called a small function with respect to $f(z)$. We shall call a small function $a(z)$ of a Nevanlinna deficient function of $f(z)$ if and only if

$$
\delta(a(z), f)=\liminf _{r \rightarrow \infty} \frac{m\left(r, \frac{1}{f(z)-a(z)}\right)}{T(r, f)}=1-\limsup _{r \rightarrow \infty} \frac{N(r, a(z), f)}{T(r, f)}>0 .
$$

\section{Communicated by James K. Langley.}

$\bowtie \quad \mathrm{Nan} \mathrm{Wu}$

wunan2007@163.com

1 Department of Mathematics, School of Science, China University of Mining and Technology (Beijing), Beijing 100083, People's Republic of China 
Recall the Valiron deficiency

$$
\begin{aligned}
\Delta(a(z), f) & =\limsup _{r \rightarrow \infty} \frac{m\left(r, \frac{1}{f(z)-a(z)}\right)}{T(r, f)} \\
& =1-\liminf _{r \rightarrow \infty} \frac{N(r, a(z), f)}{T(r, f)}>0 .
\end{aligned}
$$

Throughout the paper, we shall adopt the standard notation used in Nevanlinna theory (see [6]). Suppose that $f$ is a meromorphic function, $D=D\left(\alpha_{1}, \ldots, \alpha_{n}\right)$ is a system of rays

$$
\begin{aligned}
& D=\cup_{j=1}^{n}\left\{z: \arg z=\alpha_{j}\right\}, \quad \alpha_{1}<\alpha_{2}<\cdots \alpha_{n+1}=\alpha_{1}+2 \pi, \\
& \omega=\omega(D)=\max \left\{\pi /\left(\alpha_{j+1}-\alpha_{j}\right), 1 \leq j \leq n\right\},
\end{aligned}
$$

and

$$
G(\varepsilon, D)=\mathbb{C} \backslash \cup_{j=1}^{n}\left\{z:\left|\arg z-\alpha_{j}\right|<\varepsilon\right\} .
$$

We say that the $a$-points of $f$ are attracted to $D$ if for any $\varepsilon>0$,

$$
n(r, a, D, \varepsilon, f)=o(T(r, f)), \quad r \rightarrow \infty,
$$

where $n(r, a, D, \varepsilon, f)$ denotes the number of zeros of $f(z)-a(z)$ in the region $G(\varepsilon, D)$.

In 1992, Yang and Li [14] considered the distribution of arguments of the $a(z)$ points of $f(z)$ for a small function $a(z)$ with respect to $f(z)$ following Goldberg's work [4] and gave the following result.

Theorem A ([14]). Suppose that D is some system of rays and $f$ is a meromorphic function of finite order $\lambda>\omega$. If $\Delta(b(z), f)=0$ and $b(z)$-points of $f$ are attracted to $D$ for a small function $b(z)$, then $\delta(a(z), f)=0$ for any small function $a(z)$.

In fact, the work of Yang and Li [14] stems from the study of the growth of meromorphic functions with radially distributed values. A value on the extended complex plane $\widehat{\mathbb{C}}=\mathbb{C} \cup\{\infty\}$ is called a radially distributed value of a transcendental meromorphic function if most of the points at which the value is assumed to be distributed lie closely along a finite number of rays from the origin. Further, we recall the results obtained by $\mathrm{Wu}$ [12] and Zheng [15].

In 1993, Wu [12] used the Nevanlinna theory of meromorphic functions in angular domains to study this problem and obtained the following theorem.

Theorem B ([12]). Let $f(z)$ be a meromorphic function of finite lower order $\mu$ in $\mathbb{C}$. Suppose that $\arg z=\theta_{j}\left(j=1,2, \ldots, q ; 1 \leq q<\infty ; 0 \leq \theta_{1}<\theta_{2}<\cdots<\theta_{q}<\right.$ $\left.2 \pi, \theta_{q+1}=\theta_{1}+2 \pi\right)$ are $q$ rays in $\mathbb{C}$, such that for any $\varepsilon>0$ and $x=0, \infty$, we have

$$
\limsup _{r \rightarrow 1-} \frac{\log n\left(r, \cup_{j=1}^{q} \Omega\left(\theta_{j}+\varepsilon, \theta_{j+1}-\varepsilon\right), f=x\right)}{\log r} \leq \rho<\infty,
$$


where $\rho$ is a positive number, $\Omega\left(\theta_{j}+\varepsilon, \theta_{j+1}-\varepsilon\right)=\left\{z: \theta_{j}+\varepsilon<\arg z<\theta_{j+1}-\varepsilon\right\}$, $n(r, X, f=x)$ denotes the number of roots of $f(z)=x$ in the region $X \cap\{|z| \leq$ $r\}(X \subset \mathbb{C})$, counting multiplicities. If $f^{(l)}$ has a finite and non-zero deficient value $a$, then the order of $f(z)$ satisfies

$$
\lambda(f) \leq \max \left(\frac{\pi}{\omega}, \rho\right)
$$

where $\omega=\min _{1 \leq j \leq q}\left(\theta_{j+1}-\theta_{j}\right)$.

In 2003, Zheng [15] gave a simple and elementary way to study the growth of transcendental meromorphic functions in terms of their orders when they and their derivatives have radially distributed values, and obtained the following theorem.

Theorem C ([15]). Let $f(z)$ be a transcendental meromorphic function of finite lower order $\mu$ in $\mathbb{C}$ such that for some $a \in \widehat{\mathbb{C}}$ and an integer $p \geq 0, \delta=\delta\left(a, f^{(p)}\right)>0$. If for $q$ pairs $\left\{\alpha_{j}, \beta_{j}\right\}$ of real numbers satisfying

$$
-\pi \leq \alpha_{1}<\beta_{1}<\alpha_{2}<\beta_{2}<\cdots<\alpha_{q}<\beta_{q} \leq \pi
$$

and an integer $k>0$, we have

$$
\limsup _{r \rightarrow \infty} \frac{\log \left[n(r, Y, f=0)+n\left(r, Y, f^{(k)}=1\right)\right]}{\log r} \leq \rho,
$$

for $Y=\bigcup_{j=1}^{q}\left\{z: \alpha_{j} \leq \arg z \leq \beta_{j}\right\}$ and

$$
\sum_{j=1}^{q}\left(\alpha_{j+1}-\beta_{j}\right)<\frac{4}{\beta} \arcsin \sqrt{\frac{\delta}{2}}, \quad \alpha_{q+1}=\alpha_{1}+2 \pi,
$$

where $\beta=\max \{\rho, \omega, \mu\}, \omega=\max _{1 \leq j \leq q}\left\{\pi /\left(\beta_{j}-\alpha_{j}\right)\right\}$, then $\lambda(f) \leq \max \{\omega, \rho\}$.

Every angle $\left\{z: \beta_{j}<\arg z<\alpha_{j+1}\right\}(j=1,2, \ldots, q)$ is called an insignificant angle. Every angle $\left\{z: \alpha_{j}<\arg z<\beta_{j}\right\}(j=1,2, \ldots, q)$ is called a significant angle. It is easy to see that Theorem $\mathrm{C}$ is more general than Theorem $\mathrm{B}$, because the insignificant angle in Theorem B is $\cup_{j=1}^{q} \Omega\left(\theta_{j}-\varepsilon, \theta_{j}+\varepsilon\right)$.

The purpose of this paper is to extend Theorem A to the case of holomorphic curves.

\section{Notation and Main Results}

First, we give some notation for the holomorphic curve; for more details, see $[9,10]$. We denote the complex projective $n$-space by $P_{n} \mathbb{C}$ and the dual complex projective $n$-space by $\left(P_{n} \mathbb{C}\right)^{*}$. Let $G: \mathbb{C} \rightarrow P_{n} \mathbb{C}$ be a holomorphic curve and $\vec{G}=\left(g_{0}, g_{1}, \ldots, g_{n}\right)$ : $\mathbb{C} \rightarrow \mathbb{C}^{n+1} \backslash\{0\}$ its reduced representation, i.e., the entire functions

$$
g_{0}(z), g_{1}(z), \ldots, g_{n}(z)
$$


have no common zeros. We define the characteristic function $T(r, G)$ of $G$ by

$$
T(r, G)=\frac{1}{2 \pi} \int_{0}^{2 \pi} \log \left\|\vec{G}\left(r e^{i \theta}\right)\right\| \mathrm{d} \theta-\log \|\vec{G}(0)\|,
$$

where $\|\vec{G}(z)\|=\left(\sum_{j=0}^{n}\left|g_{j}(z)\right|^{2}\right)^{1 / 2}$. The order and lower order of a holomorphic curve $G(z)$ are defined as

$$
\lambda(G):=\limsup _{r \rightarrow \infty} \frac{\log T(r, G)}{\log r} ; \quad \mu(G):=\liminf _{r \rightarrow \infty} \frac{\log T(r, G)}{\log r} .
$$

For a holomorphic curve $a: \mathbb{C} \rightarrow\left(P_{n} \mathbb{C}\right)^{*}$ and $\vec{a}=\left(a_{0}, a_{1}, \ldots, a_{n}\right): \mathbb{C} \rightarrow$ $\mathbb{C}^{n+1} \backslash\{0\}$ its reduced representation, $\vec{G}(z) \cdot \vec{a}(z)$ is said to be free if $\vec{G}(z) \cdot \vec{a}(z) \neq \equiv$. Let $n(r, a, G)$ denote the number of zeros (counting multiplicities) of the entire function

$$
F(z):=\vec{G}(z) \cdot \vec{a}(z)=\sum_{j=0}^{n} g_{j}(z) a_{j}(z) \not \equiv 0
$$

in the disk $\{|z| \leq r\}$. We denote by $N(r, a, G)$ the counting function of zeros of the entire function $F(z)$, that is, $N(r, a, G)=N(r, 0, F)$.

For the sake of simplicity, we write

$$
L(r, \theta, a, G)=\log \frac{\left\|\vec{G}\left(r e^{i \theta}\right)\right\|\left\|\vec{a}\left(r e^{i \theta}\right)\right\|}{\left|\vec{G}\left(r e^{i \theta}\right) \cdot \vec{a}\left(r e^{i \theta}\right)\right|}, \quad L(r, \theta, \vec{G})=\log \left\|\vec{G}\left(r e^{i \theta}\right)\right\|,
$$

where $\vec{G}(z)$ is a reduced representation of $G(z)$. Note that $L(r, \theta, a, G)$ is independent of the choice of the reduced representation of $G$ and $a$. From the inequality $|\vec{G} \cdot \vec{a}| \leq$ $\|\vec{G}\|\|\vec{a}\|$, we have $L(r, \theta, a, G) \geq 0$.

Under the assumption that $\vec{G}(z) \cdot \vec{a}(z) \not \equiv 0$, we define the approximating function $m(r, a, G)$ of $G$ and $a$ by

$$
m(r, a, G)=\frac{1}{2 \pi} \int_{0}^{2 \pi} L(r, \phi, a, G) \mathrm{d} \phi
$$

Put

$$
T(r, a, G)=m(r, a, G)+N(r, a, G)
$$

Applying the Jensen formula to the entire function $F(z)$, we have

$$
N(r, 0, F)=\frac{1}{2 \pi} \int_{0}^{2 \pi} \log \left|F\left(r e^{i \theta}\right)\right| \mathrm{d} \theta-\log |F(0)| .
$$


This gives

$$
\begin{aligned}
T(r, a, G) & =m(r, a, G)+N(r, a, G) \\
& =\frac{1}{2 \pi} \int_{0}^{2 \pi} \log \left(\left\|\vec{G}\left(r e^{i \phi}\right)\right\|\left\|\vec{a}\left(r e^{i \phi}\right)\right\|\right) \mathrm{d} \phi-\log |\vec{G}(0) \cdot \vec{a}(0)| \\
& =T(r, G)+T(r, a)+\log \frac{\|\vec{G}(0)\|\|\vec{a}(0)\|}{|\vec{G}(0) \cdot \vec{a}(0)|}
\end{aligned}
$$

We define the Nevanlinna deficiency $\delta(a)=\delta(a, G)$ of $a$ with respect to $G$ by

$$
\delta(a, G)=1-\limsup _{r \rightarrow \infty} \frac{N(r, a, G)}{T(r, a)+T(r, G)} .
$$

The curve $a(z)$ is called a Nevanlinna deficient curve if $\delta(a, G)>0$. We notice that if $a$ satisfies

$$
T(r, a)=o(T(r, G)), \quad r \rightarrow \infty,
$$

i.e., $a(z)$ is a small holomorphic curve of $G(z)$, then (2.4) is reduced to

$$
\delta(a, G)=1-\limsup _{r \rightarrow \infty} \frac{N(r, a, G)}{T(r, G)} .
$$

Given an angle $\Omega=\{z: \alpha<\arg z<\beta\},(0<\beta-\alpha<2 \pi)$, define the counting function of $a(z)$-points of $G(z)$ in $\Omega$ as

$$
N(r, \Omega, a, G)=\int_{0}^{r} \frac{n(t, \Omega, a, G)}{t} \mathrm{~d} t
$$

where $n(t, \Omega, a, G)$ is the number of roots of $F=\vec{G}(z) \cdot \vec{a}(z)$ in $\Omega \cap\{z:|z|<t\}$, counting multiplicities.

We consider $q$ pairs $\left\{\alpha_{j}, \beta_{j}\right\}$ of real numbers such that

$$
-\pi \leq \alpha_{1}<\beta_{1} \leq \alpha_{2}<\beta_{2} \leq \cdots \leq \alpha_{q}<\beta_{q} \leq \pi
$$

and define $\omega=\max _{1 \leq i \leq q}\left\{\pi /\left(\beta_{i}-\alpha_{i}\right)\right\}$. Using Zheng's idea [15], we prove the following theorems.

Theorem 2.1 Let $G(z): \mathbb{C} \rightarrow P_{n} \mathbb{C}$ be a holomorphic curve of finite lower order $\mu$ and let $a(z): \mathbb{C} \rightarrow\left(P_{n} \mathbb{C}\right)^{*}$ be a small holomorphic curve of $G(z)$ such that $\delta=\delta(a, G)>0$ and $\vec{G}(z) \cdot \vec{a}(z) \not \equiv 0$. If for $q$ pairs $\left\{\alpha_{j}, \beta_{j}\right\}$ of real numbers satisfying (2.7) and a holomorphic curve $b(z): \mathbb{C} \rightarrow\left(P_{n} \mathbb{C}\right)^{*}$ and some $d \geq 1$, we have 


$$
\begin{aligned}
& \frac{1}{2 \pi} \sum_{j=1}^{q} \int_{\alpha_{j}}^{\beta_{j}} L(r, \theta, b, G) \mathrm{d} \theta=o(T(d r, G)), \\
& n(r, Y, b, G)=o(T(d r, G)),
\end{aligned}
$$

for $Y=\bigcup_{j=1}^{q}\left\{z: \alpha_{j}<\arg z<\beta_{j}\right\}$ and

$$
\sum_{i=1}^{q}\left(\alpha_{i+1}-\beta_{i}\right)<\frac{4}{\beta} \arcsin \sqrt{\frac{\delta}{2}}, \quad \alpha_{q+1}=2 \pi+\alpha_{1},
$$

where $\beta=\max \{\omega, \mu\}$, then $\lambda(G) \leq \omega$.

Theorem 2.2 Let $G(z)$ and $a(z), b(z)$ be given as in Theorem 2.1. If for q pairs $\left\{\alpha_{i}, \beta_{i}\right\}$ of real numbers satisfying (2.7), we have

$$
\begin{aligned}
& \limsup _{r \rightarrow \infty} \frac{\log \left(\frac{1}{2 \pi} \sum_{j=1}^{q} \int_{\alpha_{j}}^{\beta_{j}} L(r, \theta, b, G) \mathrm{d} \theta\right)}{\log r} \leq \rho_{1}, \\
& \limsup _{r \rightarrow \infty} \frac{\log (n(r, Y, b, G))}{\log r} \leq \rho_{2},
\end{aligned}
$$

for $Y=\bigcup_{j=1}^{q}\left\{z: \alpha_{j}<\arg z<\beta_{j}\right\}$ and (2.9) for $\beta=\max \left\{\omega, \rho_{1}, \rho_{2}, \rho(a)\right\}, \rho(a)$ is the order of $a(z)$, then $\lambda(G) \leq \max \left\{\omega, \rho_{1}, \rho_{2}\right\}$.

It is necessary to point out that the holormorphic curve $b(z)$ in Theorems $2.1,2.2$ and 2.3 need not be a small holormorphic curve with respect to $G(z)$.

From Theorems 2.1 and 2.2, we have an immediate consequence. We consider $q$ rays $\arg z=\alpha_{j}(j=1,2, \ldots, q)$, satisfying

$$
-\pi<\alpha_{1}<\alpha_{2}<\cdots<\alpha_{q}<\pi, \quad \alpha_{q+1}=\alpha_{1}+2 \pi,
$$

and write $\widetilde{\omega}=\max _{1 \leq i \leq q}\left\{\frac{\pi}{\alpha_{j+1}-\alpha_{j}}\right\}$.

Corollary 2.1 Let $G(z)$ and $a(z), b(z)$ be given as in Theorem 2.1. Then for $q$ rays $\arg z=\alpha_{i}(1 \leq i \leq q)$ satisfying (2.11), the following two statements hold.

(I) If for each small $\varepsilon>0$, we have (2.8) for $Y=\mathbb{C} \backslash \cup_{i=1}^{q}\left\{z:\left|\arg z-\alpha_{i}\right|<\varepsilon\right\}$, then $\lambda(G) \leq \widetilde{\omega}$.

(II) If for each small $\varepsilon>0$, we have (2.10) for $Y=\mathbb{C} \backslash \cup_{i=1}^{q}\left\{z:\left|\arg z-\alpha_{i}\right|<\varepsilon\right\}$, then $\lambda(G) \leq \max \left\{\widetilde{\omega}, \rho_{1}, \rho_{2}\right\}$. 
From Theorem 2.1, we also have the following corollary.

Corollary 2.2 Let $G(z): \mathbb{C} \rightarrow P_{n} \mathbb{C}$ be a holomorphic curve of lower order $\mu$ satisfying $0<\mu<\infty$ and let $a(z): \mathbb{C} \rightarrow\left(P_{n} \mathbb{C}\right)^{*}$ be a small holomorphic curve of $G(z)$ such that $\delta=\delta(a, G)>0$ and $\vec{G}(z) \cdot \vec{a}(z) \not \equiv 0$. If for each angular domain $X=\{z: \alpha<\arg z<\beta\},(0<\beta-\alpha \leq 2 \pi)$, satisfying

$$
\beta-\alpha>\max \left\{\frac{\pi}{\mu}, 2 \pi-\frac{4}{\mu} \arcsin \sqrt{\frac{\delta}{2}}\right\}
$$

and each holomorphic curve $b(z): \mathbb{C} \rightarrow\left(P_{n} \mathbb{C}\right)^{*}$, we have

$$
\frac{1}{2 \pi} \int_{\alpha}^{\beta} L(r, \theta, b, G) \mathrm{d} \theta=o(T(r, G)),
$$

then there exists a ray $\arg z=\theta \in(\alpha, \beta)$, such that for each small $\varepsilon>0$, we have

$$
\limsup _{r \rightarrow \infty} \frac{n\left(r, Z_{\varepsilon}(\theta), b, G\right)}{T(d r, G)}>0
$$

where $Z_{\varepsilon}(\theta)=\{z: \theta-\varepsilon<\arg z<\theta+\varepsilon\}$ and $d \geq 1$.

If we remove the condition $\mu(G)<\infty$ in Theorem 2.1, then we have the following theorem.

Theorem 2.3 Let $G(z): \mathbb{C} \rightarrow P_{n} \mathbb{C}$ a holomorphic curve and let $a(z): \mathbb{C} \rightarrow\left(P_{n} \mathbb{C}\right)^{*}$ be a small holomorphic curve of $G(z)$ such that $\delta=\delta(a, G)>0$ and $\vec{G}(z) \cdot \vec{a}(z) \not \equiv 0$. Assume that $q$ rays $\arg z=\alpha_{j}(1 \leq j \leq q)$ satisfy (2.11). If there exists a holomorphic curve $b(z): \mathbb{C} \rightarrow\left(P_{n} \mathbb{C}\right)^{*}$ such that (2.10) holds for $Y=\mathbb{C} \backslash \bigcup_{j=1}^{q}\left\{z: \arg z=\alpha_{j}\right\}$, then $\lambda(G) \leq \max \{\widetilde{\omega}, \rho\}$.

\section{Nevanlinna theory of a holomorphic curve in an angle}

In this section, we generalize the Nevanlinna theory of a meromorphic function in an angle to a holomorphic curve in an angle. For the sake of simplicity, throughout this paper, we denote by $\Omega(\alpha, \beta)=\{\alpha<\arg z<\beta\}$ and by $\bar{\Omega}(\alpha, \beta)$ the closed angle. Let $G(z), a(z)$ be two holomorphic curves with reduced representation $\vec{G}(z), \vec{a}(z)$ on the angle $\bar{\Omega}=\{z: \alpha \leq \arg z \leq \beta\}$, where $0<\beta-\alpha \leq 2 \pi$. Following [5], we define the following terms 


$$
\begin{aligned}
A_{\alpha, \beta}(r, a, G) & =\frac{\omega}{\pi} \int_{1}^{r}\left(\frac{1}{t^{\omega}}-\frac{t^{\omega}}{r^{2 \omega}}\right)[L(t, \alpha, a, G)+L(t, \beta, a, G)] \frac{\mathrm{d} t}{t}, \\
B_{\alpha, \beta}(r, a, G) & =\frac{2 \omega}{\pi r^{\omega}} \int_{\alpha}^{\beta} L(r, \theta, a, G) \sin \omega(\theta-\alpha) \mathrm{d} \theta, \\
C_{\alpha, \beta}(r, a, G) & =2 \sum_{1<\left|b_{n}\right|<r}\left(\frac{1}{\left|b_{n}\right|^{\omega}}-\frac{\left|b_{n}\right|^{\omega}}{r^{2 \omega}}\right) \sin \omega\left(\theta_{n}-\alpha\right), \\
A_{\alpha, \beta}(r, \vec{G}) & =\frac{\omega}{\pi} \int_{1}^{r}\left(\frac{1}{t^{\omega}}-\frac{t^{\omega}}{r^{2 \omega}}\right)[L(r, \alpha, \vec{G})+L(r, \beta, \vec{G})] \frac{\mathrm{d} t}{t}, \\
B_{\alpha, \beta}(r, \vec{G}) & =\frac{2 \omega}{\pi r^{\omega}} \int_{\alpha}^{\beta} L(r, \theta, \vec{G}) \sin \omega(\theta-\alpha) \mathrm{d} \theta,
\end{aligned}
$$

where $\omega=\pi /(\beta-\alpha)$, and $b_{n}=\left|b_{n}\right| e^{i \theta_{n}}$ are the roots of $F(z)=\vec{G}(z) \cdot \vec{a}(z)$ on $\Omega(\alpha, \beta, r)$, appearing according to their multiplicities, and $\bar{C}_{\alpha, \beta}(r, a, G)$ is defined by the same formula as that of $C_{\alpha, \beta}(r, a, G)$ with each root appearing only once. Put

$$
S_{\alpha, \beta}(r, a, G)=A_{\alpha, \beta}(r, a, G)+B_{\alpha, \beta}(r, a, G)+C_{\alpha, \beta}(r, a, G) .
$$

We define the Nevanlinna's angular characteristic of a holomorphic curve $G$ as follows:

$$
S_{\alpha, \beta}(r, G)=A_{\alpha, \beta}(r, \vec{G})+B_{\alpha, \beta}(r, \vec{G})+O(1)
$$

where $\vec{G}(z)$ is a reduced representation of $G$. We can see that $S_{\alpha, \beta}(r, G)$ is independent of the choice of the reduced representation of $G$. We explain this as follows. If $\vec{G}=$ $\left(g_{0}, g_{1}, \ldots, g_{n}\right)$ and $\widetilde{\vec{G}}=\left(\widetilde{g_{0}}, \widetilde{g_{1}}, \ldots, \widetilde{g_{n}}\right)$ are two different reduced representations of $G$, then $\left(\widetilde{g_{0}}, \widetilde{g_{1}}, \cdots, \widetilde{g_{n}}\right)=\left(l_{0}, l g_{1}, \ldots, l g_{n}\right)$, i. e., $\widetilde{\vec{G}}=l \vec{G}$, where $l(z)$ is an entire function with no zeros. From the definition of $A_{\alpha, \beta}(r, \vec{G})$ and $B_{\alpha, \beta}(r, \vec{G})$, it follows that

$$
\begin{aligned}
& A_{\alpha, \beta}(r, \widetilde{\vec{G}})+B_{\alpha, \beta}(r, \widetilde{\vec{G}})-A_{\alpha, \beta}(r, \vec{G})-B_{\alpha, \beta}(r, \vec{G}) \\
& =\frac{\omega}{\pi} \int_{1}^{r}\left(\frac{1}{t^{\omega}}-\frac{t^{\omega}}{r^{2 \omega}}\right)\left[\log \left|l\left(t e^{i \alpha}\right)\right|+\left|l\left(t e^{i \beta}\right)\right|\right] \frac{\mathrm{d} t}{t} \\
& \quad+\frac{2 \omega}{\pi r^{\omega}} \int_{\alpha}^{\beta} \log \left|l\left(r e^{i \theta}\right)\right| \sin \omega(\theta-\alpha) \mathrm{d} \theta \\
& =A_{\alpha, \beta}(r, l)-A_{\alpha, \beta}\left(r, \frac{1}{l}\right)+B_{\alpha, \beta}(r, l)-B_{\alpha, \beta}\left(r, \frac{1}{l}\right) \\
& =S_{\alpha, \beta}(r, l)-S_{\alpha, \beta}\left(r, \frac{1}{l}\right)=O(1) .
\end{aligned}
$$

Therefore, by definition (3.2), we see that $S_{\alpha, \beta}(r, G)$ is independent of the choice of the reduced representation of $G$. 
Applying Nevanlinna's first fundamental theorem for an angle [5] to the entire function $F(z)=\vec{G}(z) \cdot \vec{a}(z)$, we have

$$
S_{\alpha, \beta}(r, 0, F)=S_{\alpha, \beta}(r, \infty, F)+O(1)
$$

That is

$$
\begin{aligned}
C_{\alpha, \beta}(r, 0, F)= & \frac{\omega}{\pi} \int_{1}^{r}\left(\frac{1}{t^{\omega}}-\frac{t^{\omega}}{r^{2 \omega}}\right)\left[\log \left|F\left(r e^{i \alpha}\right)\right|+\log \left|F\left(r e^{i \beta}\right)\right|\right] \frac{\mathrm{d} t}{t} \\
& +\frac{2 \omega}{\pi r^{\omega}} \int_{\alpha}^{\beta} \log \left|F\left(r e^{i \theta}\right)\right| \sin \omega(\theta-\alpha) \mathrm{d} \theta+O(1),
\end{aligned}
$$

where $S_{\alpha, \beta}(r, 0, F), \quad S_{\alpha, \beta}(r, \infty, F)$ and $C_{\alpha, \beta}(r, 0, F)$ are the notations of Nevannlina's theory for meromorphic functions in an angle (for details, see [5]). This gives

$$
\begin{aligned}
S_{\alpha, \beta}(r, a, G)= & (A+B)_{\alpha, \beta}(r, a, G)+C_{\alpha, \beta}(r, a, G) \\
= & \frac{\omega}{\pi} \int_{1}^{r}\left(\frac{1}{t^{\omega}}-\frac{t^{\omega}}{r^{2 \omega}}\right) \\
& \times\left[\log \left\|\vec{G}\left(r e^{i \alpha}\right)\right\|\left\|\vec{a}\left(r e^{i \alpha}\right)\right\|+\log \left\|\vec{G}\left(r e^{i \beta}\right)\right\|\left\|\vec{a}\left(r e^{i \beta}\right)\right\|\right] \frac{\mathrm{d} t}{t} \\
& +\frac{2 \omega}{\pi r^{\omega}} \int_{\alpha}^{\beta} \log \left(\left\|\vec{G}\left(r e^{i \theta}\right)\right\|\left\|\vec{a}\left(r e^{i \theta}\right)\right\|\right) \sin \omega(\theta-\alpha) \mathrm{d} \theta+O(1) \\
= & S_{\alpha, \beta}(r, G)+S_{\alpha, \beta}(r, a)+O(1) .
\end{aligned}
$$

We point out that (3.5) is the generalized Nevanlinna's first fundamental theorem in an angle for holomorphic curves.

\section{Some Lemmas}

The following result was obtained in [16] for a meromorphic function, modifying a well-known result of Yang Lo.

Lemma 4.1 ([16]) Let $G(z): \mathbb{C} \rightarrow P_{n} \mathbb{C}$ be a holomorphic curve of finite lower order $0 \leq \mu<\infty$ and order $0<\lambda \leq \infty$. Then for any finite and positive number $\beta$ satisfying $\mu \leq \beta \leq \lambda$ and any set $E$ of finite logarithmic measure, i.e., $\int_{E} t^{-1} \mathrm{~d} t<\infty$, there exists a sequence $\left\{r_{n}\right\}$ of positive numbers such that

(1) $r_{n} \notin E, \lim _{n \rightarrow \infty} \frac{r_{n}}{n}=\infty$;

(2) $\liminf _{n \rightarrow \infty} \frac{\log T\left(r_{n}, G\right)}{\log r_{n}} \geq \beta$;

(3) $T(t, G)<(1+o(1))\left(\frac{2 t}{r_{n}}\right)^{\beta} T\left(r_{n} / 2, G\right), t \in\left[r_{n} / n, n r_{n}\right]$;

(4) $T(t, G) / t^{\beta-\varepsilon_{n}} \leq 2^{\beta+1} T\left(r_{n}, G\right) / r_{n}^{\beta-\varepsilon_{n}}, 1 \leq t \leq n r_{n}, \varepsilon_{n}=[\log n]^{-2}$. 
Since the characteristic function $T(r, G)$ of a holomorphic curve $G(z)$ is also a nondecreasing, positive and continuous function defined in $(0, \infty)$, we can derive Lemma 4.1 directly from [16]. A sequence $\left\{r_{n}\right\}$ satisfying (1), (2), (3) and (4) in Lemma 4.1 is called a sequence of Pólya peaks of order $\beta$ outside $E$. Given a positive function $\Lambda(r)$ satisfying $\lim _{r \rightarrow \infty} \Lambda(r)=0$, for $r>0$, define

$$
E_{\Lambda}(r, a)=\{\theta: L(r, \theta, a, G)>\Lambda(r) T(r, G)\}
$$

Put $\sigma_{\Lambda}(a)=\liminf _{n \rightarrow \infty}$ meas $E_{\Lambda}\left(r_{n}, a\right), \sigma(a)=\inf _{\Lambda} \sigma_{\Lambda}(a)$, where meas denotes the Lebesgue measure.

The following result is a more general version of the main result of Niino [7].

Lemma 4.2 ([7]) Let $G(z): \mathbb{C} \rightarrow P_{n} \mathbb{C}$ be a holomorphic curve of finite lower order $\mu$ and order $0<\lambda \leq \infty$ and let $a(z): \mathbb{C} \rightarrow\left(P_{n} \mathbb{C}\right)^{*}$ be a small holomorphic curve of $G(z)$ such that $\delta=\delta(a, G)>0$ and $\vec{G}(z) \cdot \vec{a}(z) \not \equiv 0$. Then for any sequence $\left\{r_{n}\right\}$ of Pólya peaks of order $\beta>0(\mu \leq \beta \leq \lambda)$ for $G(z)$ and any positive function $\Lambda(r)$ with $\Lambda(r) \rightarrow 0$ as $r \rightarrow \infty$, we have

$$
\liminf _{n \rightarrow \infty} \operatorname{meas} E_{\Lambda}\left(r_{n}, a\right) \geq \min \left\{2 \pi, \frac{4}{\beta} \arcsin \sqrt{\frac{\delta}{2}}\right\} .
$$

Lemma 4.2 is called the spread relation of holomorphic curves, and was obtained in [7] for Pólya peaks of order $\mu$. The same argument of [7] can be used to derive Lemma 4.2 for Pólya peaks of order $\beta, \mu \leq \beta \leq \lambda$.

Lemma 4.3 Let $G(z): \mathbb{C} \rightarrow P_{n} \mathbb{C}$ and $a(z), b(z): \mathbb{C} \rightarrow\left(P_{n} \mathbb{C}\right)^{*}$ be three holomorphic curves. Then, for any angle $\Omega=\{z: \alpha<\arg z<\beta\},(0<\beta-\alpha \leq 2 \pi)$, any $0<\varepsilon<\frac{\beta-\alpha}{2}$, we have

$$
\begin{aligned}
\frac{1}{2 \pi} \int_{\alpha+\varepsilon}^{\beta-\varepsilon} L(r, \theta, a, G) \mathrm{d} \theta \leq & \frac{\omega_{1}}{\pi \sin (\varepsilon / 4)} \int_{\alpha+\varepsilon / 4}^{\beta-\varepsilon / 4}[L(r, \theta, b, G)+L(r, \theta, \vec{a})] \mathrm{d} \theta \\
& +\frac{2 \omega_{1}}{\pi \varepsilon \sin (\varepsilon / 4)} \int_{1}^{r}\left(\frac{r}{t}\right)^{\omega_{1}} \frac{\mathrm{d} t}{t} \\
& \times \int_{[\alpha+\varepsilon / 4, \alpha+\varepsilon / 2] \cup[\beta-\varepsilon / 2, \beta-\varepsilon / 4]} \\
& {[L(t, \theta, b, G)+L(t, \theta, \vec{a})] \mathrm{d} \theta } \\
& +\frac{1}{\sin (\varepsilon / 4)} n(r, \Omega, b, G)+\frac{\omega_{1}}{\sin (\varepsilon / 4)} \\
& \times \int_{1}^{r}\left(\frac{r}{t}\right)^{\omega_{1}} n(t, \Omega, b, G) \frac{\mathrm{d} t}{t}+O\left(r^{\omega_{1}}\right),
\end{aligned}
$$

where $\omega_{1}=\frac{\pi}{\beta-\alpha-\varepsilon}$ and $\vec{a}$ is a reduced representation of $a$. 
Proof For $\varphi \in(\varepsilon / 4, \varepsilon / 2)$, put $\omega=\frac{\pi}{\beta-\alpha-2 \varphi}$. For $\varphi \in(\varepsilon / 4, \varepsilon / 2)$ and $\theta \in(\alpha+\varepsilon, \beta-\varepsilon)$, we have the following two estimates

$$
\begin{aligned}
\omega(\theta-\alpha-\varphi) & =\frac{\pi(\theta-\alpha-\varphi)}{\beta-\alpha-2 \varphi} \geq \frac{\pi(\varepsilon-\varphi)}{\beta-\alpha-2 \varphi} \\
& \geq \frac{\pi \varepsilon / 2}{\beta-\alpha-\varepsilon / 2}>\frac{\varepsilon}{4} ; \\
\omega(\theta-\alpha-\varphi) & =\frac{\pi(\theta-\alpha-\varphi)}{\beta-\alpha-2 \varphi} \leq \frac{\pi(\beta-\alpha-\varepsilon-\varepsilon / 4)}{\beta-\alpha-\varepsilon} \\
& \leq \pi-\omega \frac{\varepsilon}{4}<\pi-\frac{\varepsilon}{4} .
\end{aligned}
$$

Therefore,

$$
\sin \omega(\theta-\alpha-\varphi) \geq \sin (\varepsilon / 4) .
$$

It follows from the definition of $B_{\alpha+\varphi, \beta-\varphi}(r, a, G)$ that

$$
\begin{aligned}
B_{\alpha+\varphi, \beta-\varphi}(r, a, G) & =\frac{2 \omega}{\pi r^{\omega}} \int_{\alpha+\varphi}^{\beta-\varphi} L(r, \theta, a, G) \sin \omega(\theta-\alpha-\varphi) \mathrm{d} \theta \\
& \geq \frac{2 \omega \sin (\varepsilon / 4)}{\pi r^{\omega}} \int_{\alpha+\varepsilon}^{\beta-\varepsilon} L(r, \theta, a, G) \mathrm{d} \theta \\
& >\frac{\sin (\varepsilon / 4)}{\pi r^{\omega}} \int_{\alpha+\varepsilon}^{\beta-\varepsilon} L(r, \theta, a, G) \mathrm{d} \theta
\end{aligned}
$$

Combining (3.5) with (4.3), and in view of (3.5), we have

$$
\begin{aligned}
\frac{\sin (\varepsilon / 4)}{\pi r^{\omega}} \int_{\alpha+\varepsilon}^{\beta-\varepsilon} L(r, \theta, a, G) \mathrm{d} \theta & \leq B_{\alpha+\varphi, \beta-\varphi}(r, a, G) \leq S_{\alpha+\varphi, \beta-\varphi}(r, a, G) \\
& =S_{\alpha+\varphi, \beta-\varphi}(r, G)+S_{\alpha+\varphi, \beta-\varphi}(r, a)+O(1) \\
& \leq S_{\alpha+\varphi, \beta-\varphi}(r, b, G)+S_{\alpha+\varphi, \beta-\varphi}(r, a)+O(1)
\end{aligned}
$$

For the sake of simplicity, we write

$$
T_{\alpha+\varphi, \beta-\varphi}(r):=T_{\alpha+\varphi, \beta-\varphi}(r, b, G)+T_{\alpha+\varphi, \beta-\varphi}(r, \vec{a}),
$$

where $T$ can stand for either of $S, A, B$. It is easy to see that

$$
\begin{aligned}
S_{\alpha+\varphi, \beta-\varphi}(r) & =A_{\alpha+\varphi, \beta-\varphi}(r)+B_{\alpha+\varphi, \beta-\varphi}(r)+C_{\alpha+\varphi, \beta-\varphi}(r, b, G), \\
r^{\omega} B_{\alpha+\varphi, \beta-\varphi}(r) & \leq \frac{2 \omega}{\pi} \int_{\alpha+\varepsilon / 4}^{\beta-\varepsilon / 4}[L(r, \theta, b, G)+L(r, \theta, \vec{a})] \mathrm{d} \theta .
\end{aligned}
$$


By the definition of $C_{\alpha, \beta}(r, *)$, it follows that

$$
r^{\omega} C_{\alpha+\varphi, \beta-\varphi}(r, *) \leq 2 n(r, \Omega, *)+2 \omega \int_{1}^{r}\left(\frac{r}{t}\right)^{\omega} n(t, \Omega, *) \frac{\mathrm{d} t}{t} .
$$

Inequality (4.4) yields

$$
\int_{\alpha+\varepsilon}^{\beta-\varepsilon} L(r, \theta, a, G) \mathrm{d} \theta \leq\left[S_{\alpha+\varphi, \beta-\varphi}(r)+O(1)\right] \frac{\pi r^{\omega}}{\sin (\varepsilon / 4)} .
$$

By a simple calculation, we get

$$
\begin{aligned}
r^{\omega} \int_{\varepsilon / 4}^{\varepsilon / 2} A_{\alpha+\varphi, \beta-\varphi}(r) \mathrm{d} \varphi & \leq \frac{\omega_{1}}{\pi} \int_{1}^{r} \frac{\mathrm{d} t}{t} \int_{[\alpha+\varepsilon / 4, \alpha+\varepsilon / 2] \cup[\beta-\varepsilon / 2, \beta-\varepsilon / 4]} \\
& \times\left[\left(\frac{r}{t}\right)^{\omega}-\left(\frac{t}{r}\right)^{\omega}\right][L(t, \varphi, b, G)+L(r, \varphi, \vec{a})] \mathrm{d} \varphi \\
\leq & \frac{\omega_{1}}{\pi} \int_{1}^{r}\left(\frac{r}{t}\right)^{\omega_{1}} \frac{\mathrm{d} t}{t} \int_{[\alpha+\varepsilon / 4, \alpha+\varepsilon / 2] \cup[\beta-\varepsilon / 2, \beta-\varepsilon / 4]} \\
& \times[L(t, \varphi, b, G)+L(r, \varphi, \vec{a})] \mathrm{d} \varphi .
\end{aligned}
$$

Integrating (4.8) for $\varphi \in[\varepsilon / 4, \varepsilon / 2]$ and combining (4.6), (4.7) with (4.9), we have

$$
\begin{aligned}
\varepsilon \int_{\alpha+\varepsilon}^{\beta-\varepsilon} L(r, \theta, a, G) \mathrm{d} \theta \leq & \frac{2 \omega_{1} \varepsilon}{\sin (\varepsilon / 4)} \int_{\alpha+\varepsilon / 4}^{\beta-\varepsilon / 4}[L(r, \theta, b, G)+L(r, \theta, \vec{a})] \mathrm{d} \theta \\
& +\frac{4 \omega_{1}}{\sin (\varepsilon / 4)} \int_{1}^{r}\left(\frac{r}{t}\right)^{\omega_{1}} \frac{\mathrm{d} t}{t} \int_{[\alpha+\varepsilon / 4, \alpha+\varepsilon / 2] \cup[\beta-\varepsilon / 2, \beta-\varepsilon / 4]} \\
& \times[L(t, \varphi, b, G)+L(r, \varphi, \vec{a})] \mathrm{d} \varphi \\
& +\frac{2 \pi \varepsilon}{\sin (\varepsilon / 4)} n(r, \Omega, b, G)+\frac{2 \pi \omega_{1} \varepsilon}{\sin (\varepsilon / 4)} \\
& \int_{1}^{r}\left(\frac{r}{t}\right)^{\omega_{1}} n(t, \Omega, b, G) \frac{\mathrm{d} t}{t}+O\left(r^{\omega_{1}}\right) .
\end{aligned}
$$

The proof is complete.

Lemma 4.4 Let $G(z): \mathbb{C} \rightarrow P_{n} \mathbb{C}$ and $a(z), b(z): \mathbb{C} \rightarrow\left(P_{n} \mathbb{C}\right)^{*}$ be three holomorphic curves. If for an angle $\Omega=\{z: \alpha<\arg z<\beta\},(0<\beta-\alpha \leq 2 \pi)$ and some $p \geq 1$ and a reduced representation $\vec{a}$ of $a$, we have

$$
\begin{aligned}
& \frac{1}{2 \pi} \int_{\alpha}^{\beta}[L(r, \theta, b, G)+L(r, \theta, \vec{a})] \mathrm{d} \theta=o(T(p r, G)), \\
& n(r, \Omega, b, G)=o(T(p r, G)),
\end{aligned}
$$


then for any $0<\varepsilon<\frac{\beta-\alpha}{2}$, any sequence of Pólya peaks $\left\{r_{n}\right\}$ oforder $\sigma>\omega_{1}=\frac{\pi}{\beta-\alpha-\varepsilon}$ for $T(r, G)$, we have

$$
\frac{1}{2 \pi} \int_{\alpha+\varepsilon}^{\beta-\varepsilon} L\left(r_{n}, \theta, a, G\right) \mathrm{d} \theta=o\left(T\left(r_{n}, G\right)\right)
$$

Proof As $\left\{r_{n}\right\}$ is a sequence of Pólya peaks of order $\sigma>\omega_{1}$ for $T(r, G)$, for any real function $l(t)$ satisfying that $l(t)=o(T(p t, G))(p \geq 1)$, we have

$$
\begin{aligned}
\int_{1}^{r_{n}}\left(\frac{r_{n}}{t}\right)^{\omega_{1}} l(t) \frac{\mathrm{d} t}{t} & =o\left(\int_{1}^{r_{n}}\left(\frac{r_{n}}{t}\right)^{\omega_{1}} T(p t, G) \frac{\mathrm{d} t}{t}\right) \\
& \leq o\left(T\left(r_{n}, G\right) \int_{1}^{r_{n}}\left(\frac{r_{n}}{t}\right)^{\omega_{1}}\left(\frac{p t}{r_{n}}\right)^{\sigma-\varepsilon_{n}} \frac{\mathrm{d} t}{t}\right) \\
& =o\left(T\left(r_{n}, G\right)\right) .
\end{aligned}
$$

Substituting $l(t)=n(t, \Omega, b, G)$ and $l(t)=\int_{\alpha}^{\beta}[L(t, \theta, b, G)+L(t, \theta, \vec{a})] \mathrm{d} \theta$ to (4.2), we get the result.

Lemma 4.5 Let $G(z): \mathbb{C} \rightarrow P_{n} \mathbb{C}$ and $a(z), b(z): \mathbb{C} \rightarrow\left(P_{n} \mathbb{C}\right)^{*}$ be three holomorphic curves. If for an angle $\Omega=\{z: \alpha<\arg z<\beta\},(0<\beta-\alpha \leq 2 \pi)$, we have

$$
\limsup _{r \rightarrow \infty} \frac{\log \left(\frac{1}{2 \pi} \int_{\alpha}^{\beta} L(r, \theta, b, G) \mathrm{d} \theta\right)}{\log r} \leq \rho_{1}, \quad \limsup _{r \rightarrow \infty} \frac{\log n(r, \Omega, b, G)}{\log r} \leq \rho_{2}
$$

then for any $0<\varepsilon<\frac{\beta-\alpha}{2}$, we have

$$
\frac{1}{2 \pi} \int_{\alpha+\varepsilon}^{\beta-\varepsilon} L(r, \theta, a, G) \mathrm{d} \theta=O\left(r^{\eta+\varepsilon}\right)
$$

where $\eta=\max \left\{\rho_{1}, \rho_{2}, \omega_{1}, \rho(a)\right\}, \omega_{1}=\pi /(\beta-\alpha-\varepsilon)$.

Proof Since

$$
\limsup _{r \rightarrow \infty} \frac{\log \left(\frac{1}{2 \pi} \int_{\alpha}^{\beta} L(r, \theta, \vec{a}) \mathrm{d} \theta\right)}{\log r} \leq \rho(a)
$$

then

$$
\int_{\alpha}^{\beta}[L(r, \theta, b, G)+L(r, \theta, \vec{a})] \mathrm{d} \theta=O\left(r^{\max \left\{\rho_{1}, \rho(a)\right\}+\varepsilon}\right) .
$$


For any real function $l(t)$ satisfying $l(t)=O\left(t^{\rho+\varepsilon}\right)$, we have

$$
\begin{aligned}
\int_{1}^{r}\left(\frac{r}{t}\right)^{\omega_{1}} l(t) \frac{\mathrm{d} t}{t} & =O\left(\int_{1}^{r_{n}}\left(\frac{r}{t}\right)^{\omega_{1}} t^{\rho+\varepsilon} \frac{\mathrm{d} t}{t}\right) \\
& =O\left(r^{\omega_{1}} \int_{1}^{r} t^{\rho+\varepsilon-\omega_{1}-1} \mathrm{~d} t\right) \\
& =O\left(r^{\max \left\{\rho, \omega_{1}\right\}+\varepsilon}\right) .
\end{aligned}
$$

Substituting $l(t)=\int_{\alpha}^{\beta}[L(t, \theta, b, G)+L(t, \theta, \vec{a})] \mathrm{d} \theta, \rho=\max \left\{\rho_{1}, \rho(a)\right\}$ or $l(t)=$ $n(t, \Omega, b, G), \rho=\rho_{2}$ to (4.2), we get the result.

We establish Lemma 4.6 by the Poisson-Jensen formula of meromorphic functions to estimate the logarithmic module $L(r, \theta, a, G)$.

Lemma 4.6 Let $G(\xi): \Delta \rightarrow P_{n} \mathbb{C}$ be a holomorphic curve and let $a(\xi): \Delta \rightarrow$ $\left(P_{n} \mathbb{C}\right)^{*}$ be a holomorphic curve with reduced representations $\vec{G}$ and $\vec{a}$ such that $\vec{G}(\xi) \cdot \vec{a}(\xi) \not \equiv 0$ in the unit disk. Then, for any $z=r e^{i \theta}$ such that $0<r<R<1$, we have

$$
L(r, \theta, a, G) \leq \log ^{+}(n+1)+\frac{R+r}{R-r} m(R, a, G)+\sum_{t=1}^{M} \log \left|\frac{2 R}{z-b_{t}}\right|
$$

where $b_{1}, b_{2}, \ldots, b_{M}$ are the roots of $F(\xi):=\vec{G}(\xi) \cdot \vec{a}(\xi)$ in $|\xi|<R$.

Proof We prove that (4.11) holds for every point $z$. For any $z=r e^{i \theta}, 0<r<R<1$, there exist two integers $0 \leq k=k_{z} \leq n, 0 \leq l=l_{z} \leq n$, such that

$$
\max _{0 \leq i \leq v}\left|G_{i}(z)\right|=\left|G_{k}(z)\right|, \quad \max _{0 \leq j \leq \nu}\left|a_{j}(z)\right|=\left|a_{l}(z)\right|
$$

Then, we have

$$
\begin{aligned}
\log ^{+} \frac{\|\vec{G}(z)\|\|\vec{a}(z)\|}{|F(z)|} & \leq \log ^{+} \frac{(n+1)^{1 / 2}\left|G_{k}(z)\right|(n+1)^{1 / 2}\left|a_{l}(z)\right|}{|F(z)|} \\
& \leq \log ^{+}(n+1)+\log ^{+} \frac{\left|G_{k}(z)\right|\left|a_{l}(z)\right|}{|F(z)|} \\
& =\log ^{+}(n+1)+\log ^{+}\left|\frac{G_{k}(z) a_{l}(z)}{F(z, a)}\right| .
\end{aligned}
$$

Notice that both $G_{k}(\xi) \cdot a_{l}(\xi)$ and $F(\xi)$ are entire functions, so

$$
\frac{G_{k}(\xi) \cdot a_{l}(\xi)}{F(\xi)}
$$


is a meromorphic function. We apply the Possion-Jensen formula to the meromorphic function $G_{k}(\xi) \cdot a_{l}(\xi) / F(\xi)$ to get

$$
\begin{aligned}
\log ^{+}\left|\frac{G_{k}(z) \cdot a_{l}(z)}{F(z)}\right| \leq & \frac{1}{2 \pi} \int_{0}^{2 \pi} \log ^{+}\left|\frac{G_{k}\left(R e^{i \phi}\right) a_{l}\left(R e^{i \phi}\right)}{F\left(R e^{i \phi}\right)}\right| \\
& \times \frac{R^{2}-r^{2}}{R^{2}-2 R r \cos (\theta-\phi)+r^{2}} \mathrm{~d} \phi \\
& +\sum_{t=1}^{M} \log \left|\frac{R^{2}-\bar{b}_{t} z}{R\left(z-b_{t}\right)}\right|
\end{aligned}
$$

Using the inequality $\frac{R^{2}-r^{2}}{R^{2}-2 R r \cos (\theta-\phi)+r^{2}} \leq \frac{R+r}{R-r}$, we derive

$$
\begin{aligned}
\log ^{+}\left|\frac{G_{k}(z) \cdot a_{l}(z)}{F(z)}\right| \leq & \frac{1}{2 \pi} \frac{R+r}{R-r} \int_{0}^{2 \pi} \log ^{+}\left|\frac{G_{k}\left(R e^{i \phi}\right) a_{l}\left(R e^{i \phi}\right)}{F\left(R e^{i \phi}\right)}\right| \\
& \times \mathrm{d} \phi+\sum_{t=1}^{M} \log \left|\frac{2 R}{z-b_{t}}\right|
\end{aligned}
$$

In view of $\left|G_{k}\left(R e^{i \phi}\right)\right| \leq\left\|\vec{G}\left(R e^{i \phi}\right)\right\|$ and $\left|a_{l}\left(R e^{i \phi}\right)\right| \leq\left\|\vec{a}\left(R e^{i \phi}\right)\right\|$, we have

$$
\log ^{+}\left|\frac{G_{k}(z) \cdot a_{l}(z)}{F(z)}\right| \leq \frac{R+r}{R-r} m(R, \vec{a}, \vec{A})+\sum_{t=1}^{M} \log \left|\frac{2 R}{z-b_{t}}\right|
$$

This completes the proof.

To prove Theorem 2.3, we need Lemma 4.7, which is a generalization of a result of Edrei [2].

Lemma 4.7 Let $G(z): \mathbb{C} \rightarrow P_{n} \mathbb{C}$ be a holomorphic curve and let $a(z): \mathbb{C} \rightarrow\left(P_{n} \mathbb{C}\right)^{*}$ be a small holomorphic curve of $G(z)$ such that $\vec{G}(z) \cdot \vec{a}(z) \not \equiv 0$ and $\delta=\delta(a, G)>0$. Then, given $\varepsilon>0$, we have

$$
\text { meas } E(r, a)>\frac{1}{T^{\varepsilon}(r, a, G)[\log r]^{1+\varepsilon}}, \quad r \notin F,
$$

where

$$
E(r, a)=\left\{\theta \in[-\pi, \pi): L(r, \theta, a, G)>\frac{\delta}{4} T(r, G)\right\},
$$

and $F$ is a set of positive real numbers of finite logarithmic measure depending on $\varepsilon$. Proof Let $\left\{b_{t}\right\}$ be the union of the sequence of the roots of $F(z):=\vec{G}(z) \cdot \vec{a}(z)$ and the sequence of roots of $F(z)=0$ (Assume that $\left\{\left|b_{t}\right|\right\}$ is non-decreasing and that the 
multiplicities of the roots have been taken into account by a suitable repetition of the elements). Let

$$
I_{t}:=\left(\left|b_{t}\right|-\frac{1}{t^{2}},\left|b_{t}\right|+\frac{1}{t^{2}}\right)
$$

If $r>0$ and $r \notin \bigcup_{t=1}^{\infty} I_{t}$, then combining (4.12) with (4.13), we have $(r<R)$ when

$$
\begin{aligned}
L(r, \theta, a, G) \leq & \log ^{+}(n+1)+\frac{R+r}{R-r} m(R, \vec{a}, \vec{A}) \\
& +n(R)[\log 2 R+2 \log n(R)],
\end{aligned}
$$

where $n(R)$ denotes the number of roots of $F(z)=0$ in $|z| \leq R$. Setting $R^{\prime}-R=$ $R-r$, we deduce

$$
N\left(R^{\prime}, 0, F(z)\right)-N(R, 0, F(z))=\int_{R}^{R^{\prime}} \frac{n(t) \mathrm{d} t}{t}>\frac{n(R)}{R^{\prime}} \frac{R^{\prime}-r}{2} .
$$

Substituting the above into (4.14), it follows that

$$
\begin{aligned}
L(r, \theta, a, G) \leq & \log ^{+}(n+1)+\frac{4 R^{\prime}}{R^{\prime}-r} m\left(R^{\prime}, a, G\right) \\
& +\frac{2 R^{\prime} N\left(R^{\prime}, a, G\right)}{R^{\prime}-r}\left[\log 2 R^{\prime}+2 \log \left(\frac{2 R^{\prime}}{R^{\prime}-r} N\left(R^{\prime}, a, G\right)\right)\right] \\
\leq & \log ^{+}(n+1) \\
& +\frac{4 R^{\prime}}{R^{\prime}-r} T\left(R^{\prime}, a, G\right)\left[\left(1+\frac{3}{2} \log 2\right)\right. \\
& \left.+\frac{1}{2} \log R^{\prime}+\frac{R^{\prime}}{R^{\prime}-r}+\log T\left(R^{\prime}, a, G\right)\right] .
\end{aligned}
$$

If $r$ is large enough, say $r$ is larger than some $r_{0}$, we have

$$
L(r, \theta, a, G) \leq A\left(\frac{R^{\prime}}{R^{\prime}-r}\right)^{2}\left(\log R^{\prime}\right) T\left(R^{\prime}, a, G\right) \log T\left(R^{\prime}, a, G\right)
$$

where $A$ is a positive constant depending on $v$, for $r>r_{0}, r \notin \cup I_{t}$. For $r>r_{0}$, the function

$$
V(r)=[T(r, a, G) \log T(r, a, G)] \log r
$$

is positive, continuous and non-decreasing to infinity. Hence, for any $\eta>0$, according to Borel's lemma, we have

$$
V\left(r\left(1+\frac{1}{\log V(r)}\right)\right)<V^{1+\eta}(r)
$$


except possibly for values of $r>r_{0}$ which belong to an exceptional set $\mathcal{E}(\eta)$, of finite logarithmic measure. Taking, in (4.15)

$$
R^{\prime}=r\left(1+\frac{1}{\log V(r)}\right)
$$

we obtain

$$
L(r, \theta, a, G) \leq V^{1+2 \eta}(r)
$$

provided $r$ is sufficiently large and $r \notin \mathcal{E}(\eta) \cup \bigcup_{t} I_{t}$. It is easy to see that the set $\mathcal{E}(\eta) \cup \bigcup_{t} I_{t}$ is of finite logarithmic measure. For $r \notin \mathcal{E}(\eta) \cup \bigcup_{t} I_{t}$, consider the set

$$
E=\left\{\theta \in[-\pi, \pi): L(r, \theta, a, G)>\frac{1}{2} m(r, a, G)\right\} .
$$

Then

$$
\begin{array}{r}
2 \pi m(r, a, G) \leq \int_{E} L(r, \theta, a, G) d \theta+2 \pi \frac{m(r, a, G)}{2}, \\
\pi m(r, a, G)<V^{1+2 \eta}(r) \text { meas } E .
\end{array}
$$

If $r$ is sufficiently large, we have

$$
\begin{aligned}
& m(r, a, G)>\frac{\delta}{2} T(r, a, G)=\frac{\delta}{2}(T(r, G)+T(r, a)+O(1)), \\
& E \subset E(r, a) .
\end{aligned}
$$

Therefore,

$$
\text { meas } E(r, a)>\frac{\delta / 2(T(r, G)+T(r, a)+O(1))}{T^{1+2 \eta}(r, a, G)[\log T(r, a, G)]^{1+2 \eta} \log ^{1+2 \eta} r} .
$$

Taking $\eta=\varepsilon / 3$, we obtain the result.

\section{Proof of Theorem 2.1}

The idea of the proof comes from [15]. Suppose that the theorem is not true, i.e. $\lambda(G)>\omega$. We treat it as two cases. If $\lambda(G)>\mu(G)$, then $\beta$ is between $\lambda(G)$ and $\mu(G)$. If $\lambda(G)=\mu(G)$, then $\beta=\lambda(G)=\mu(G)$. Thus, we consider the following two cases.

I. $\lambda(G)>\beta \geq \mu(G)$. By (2.9), we can choose a real number $\varepsilon>0$, such that

$$
\sum_{i=1}^{q}\left(\alpha_{i+1}-\beta_{i}+2 \varepsilon\right)+2 \varepsilon<\frac{4}{\beta+2 \varepsilon} \arcsin \sqrt{\frac{\delta}{2}}
$$


where $\alpha_{q+1}=2 \pi+\alpha_{1}$, and $\lambda(G)>\beta+2 \varepsilon>\mu$. Applying Lemma 4.2 to $G(z)$ gives the existence of a sequence $\left\{r_{n}\right\}$ of Pólya peaks of order $\beta+2 \varepsilon$ for $G(z)$. Set $\Lambda(r)=\Gamma^{1 / 2}(r)$ and

$$
\Gamma(r)=\max \left\{\frac{\frac{1}{2 \pi} \int_{\alpha_{i}+\varepsilon}^{\beta_{i}-\varepsilon} L\left(r_{n}, \theta, a, G\right) \mathrm{d} \theta}{T\left(r_{n}, G\right)} ; 1 \leq i \leq q\right\}, \quad r_{n-1}<r \leq r_{n} .
$$

Since $a(z)$ is a small holomorphic curve of $G(z)$, then

$$
\begin{aligned}
& \frac{1}{2 \pi} \sum_{j=1}^{q} \int_{\alpha_{j}}^{\beta_{j}} L(r, \theta, \vec{a}) \mathrm{d} \theta \leq T(r, a) \\
& \quad+\log \|\vec{a}(0)\|=o(T(r, G))=o(T(d r, G)) .
\end{aligned}
$$

Combining this with (2.8), we have

$$
\frac{1}{2 \pi} \sum_{j=1}^{q} \int_{\alpha_{j}}^{\beta_{j}}[L(r, \theta, b, G)+L(r, \theta, \vec{a})] \mathrm{d} \theta=o(T(d r, G))
$$

Using Lemma 4.4 to Pólya peaks $\left\{r_{n}\right\}$ of order $\beta+2 \varepsilon$ for $G(z)$ and from $\beta+2 \varepsilon>$ $\omega_{i}=\pi /\left(\beta_{i}-\alpha_{i}-\varepsilon\right)$, we can deduce that $\lim _{r \rightarrow \infty} \Lambda(r)=0$. Then from Lemma 4.2, for sufficiently large $n$, we have

$$
\text { meas } E_{\Lambda}\left(r_{n}, a\right)>\frac{4}{\beta+2 \varepsilon} \arcsin \sqrt{\frac{\delta}{2}}-\varepsilon
$$

since $\beta+2 \varepsilon>1 / 2$. We can assume (5.3) holds for all $n$. Put

$$
K:=\operatorname{meas}\left(E_{\Lambda}\left(r_{n}, a\right) \bigcap \bigcup_{i=1}^{q}\left(\alpha_{i}+\varepsilon, \beta_{i}-\varepsilon\right)\right)
$$

From (5.1) and (5.3), we derive

$$
\begin{aligned}
K & \geq \operatorname{meas}\left(E_{\Lambda}\left(r_{n}, a\right)\right)-\operatorname{meas}\left([-\pi, \pi) \backslash \bigcup_{i=1}^{q}\left(\alpha_{i}+\varepsilon, \beta_{i}-\varepsilon\right)\right) \\
& =\operatorname{meas}\left(E_{\Lambda}\left(r_{n}, a\right)\right)-\operatorname{meas}\left(\bigcup_{i=1}^{q}\left(\beta_{i}-\varepsilon, \alpha_{i+1}+\varepsilon\right)\right) \\
& =\operatorname{meas}\left(E_{\Lambda}\left(r_{n}, a\right)\right)-\sum_{i=1}^{q}\left(\alpha_{i+1}-\beta_{i}+2 \varepsilon\right)>\varepsilon>0 .
\end{aligned}
$$


It is easy to see that there exists $i_{0}$ such that for infinitely many $n$, we have

$$
\operatorname{meas}\left(E_{\Lambda}\left(r_{n}, a\right) \bigcap\left(\alpha_{i_{0}}+\varepsilon, \beta_{i_{0}}-\varepsilon\right)\right)>\frac{K}{q}>\frac{\varepsilon}{q} \text {. }
$$

We can assume (5.4) holds for all $n$. Set $E_{n}=E_{\Lambda}\left(r_{n}, a\right) \bigcap\left(\alpha_{i_{0}}+\varepsilon, \beta_{i_{0}}-\varepsilon\right)$. By the definition of $E_{\Lambda}\left(r_{n}, a\right)$, it follows that

$$
\begin{aligned}
& \int_{\alpha_{i_{0}}+\varepsilon}^{\beta_{i_{0}}-\varepsilon} L\left(r_{n}, \theta, a, G\right) d \theta \geq \int_{E_{n}} L\left(r_{n}, \theta, a, G\right) \mathrm{d} \theta \\
& \geq \operatorname{meas}\left(E_{n}\right) \Lambda\left(r_{n}\right) T\left(r_{n}, G\right) \geq \frac{\varepsilon}{q} \Lambda\left(r_{n}\right) T\left(r_{n}, G\right) .
\end{aligned}
$$

On the other hand, by (5.2), we have

$$
\int_{\alpha_{i_{0}}+\varepsilon}^{\beta_{i_{0}}-\varepsilon} L\left(r_{n}, \theta, a, G\right) \mathrm{d} \theta \leq \Lambda^{2}\left(r_{n}\right) T\left(r_{n}, G\right) .
$$

Combining (5.5) with (5.6) gives

$$
\frac{\varepsilon}{q} \leq \Lambda\left(r_{n}\right) \rightarrow 0
$$

This is impossible.

II. $\lambda(G)=\mu(G)$. Then $\beta=\mu=\lambda(G)$. By the same argument as in I, with $\beta+2 \varepsilon$ replaced by $\beta=\mu$ everywhere, we can derive a contradiction.

Theorem 2.1 follows.

\section{Proof of Theorem 2.2}

Suppose that $\lambda(G)>\max \left\{\omega, \rho_{1}, \rho_{2}\right\}$. We will derive a contradiction by making a minor modification to the proof of Theorem 2.1. We use the same notation as in Theorem 2.1 .

I. $\lambda(G)>\mu$. $\left\{r_{n}\right\}$ is a sequence of the Pólya peaks of order $\beta+2 \varepsilon$ for $G(z)$. Set $\Lambda(r)=[\log r]^{-1}$. Then we deduce that

$$
\int_{\alpha_{i_{0}}+\varepsilon}^{\beta_{i_{0}}-\varepsilon} L\left(r_{n}, \theta, a, G\right) \mathrm{d} \theta>\frac{\varepsilon}{q} \frac{T\left(r_{n}, G\right)}{\log r_{n}} .
$$

On the other hand, by Lemma 4.5 and noting that $\eta \leq \beta$, we have

$$
\int_{\alpha_{i_{0}}+\varepsilon}^{\beta_{i_{0}}-\varepsilon} L\left(r_{n}, \theta, a, G\right) \mathrm{d} \theta \leq O\left(r_{n}^{\beta+\varepsilon}\right) .
$$


Combining (6.1) with (6.2) gives

$$
T\left(r_{n}, G\right) \leq \frac{q K}{\varepsilon} r_{n}^{\beta+\varepsilon} \log r_{n}
$$

Therefore, we have

$$
\beta+2 \varepsilon \leq \frac{\log T\left(r_{n}, G\right)}{\log r_{n}} \leq \beta+\varepsilon .
$$

This leads to a contradiction.

II. $\lambda(G)=\mu$, then $\beta=\mu=\lambda(G)$. By the same arguments as in I with all the $\beta+2 \varepsilon$ replaced by $\beta=\mu$, we can derive

$$
\mu=\beta \leq \max \left\{\rho_{1}, \rho_{2}, \omega\right\}+\varepsilon<\lambda(G) .
$$

This is also impossible.

Theorem 2.2 follows.

\section{Proof of Theorem 2.3}

Applying Lemma 4.7 to $G(z)$ implies the existence of a sequence $\left\{r_{n}\right\}$ of positive numbers such that $r_{n} \rightarrow \infty$ and $r_{n} \notin F$ and

$$
\operatorname{meas} E\left(r_{n}, a\right) \geq \frac{1}{T^{\varepsilon}\left(r_{n}, a, G\right)\left[\log r_{n}\right]^{1+\varepsilon}},
$$

where $F$ is the set in Lemma 4.7. Put

$$
\varepsilon_{n}=\frac{1}{(2 q+1)} \frac{1}{T^{\varepsilon}\left(r_{n}, a, G\right)\left[\log r_{n}\right]^{1+\varepsilon}} .
$$

Then it follows from (7.1) that

$$
\begin{aligned}
& \operatorname{meas}\left(E\left(r_{n}, a\right) \bigcap \bigcup_{i=1}^{q}\left(\alpha_{i}+\varepsilon_{n}, \alpha_{i+1}-\varepsilon_{n}\right)\right) \\
& \geq \operatorname{meas} E\left(r_{n}, a\right)-\operatorname{meas}\left(\bigcup_{i=1}^{q}\left(\alpha_{i}+\varepsilon_{n}, \alpha_{i+1}-\varepsilon_{n}\right)\right) \\
& \geq(2 q+1) \varepsilon_{n}-2 q \varepsilon_{n}=\varepsilon_{n}>0 .
\end{aligned}
$$

Thus, there exists a $j$ such that for infinitely many $n$, we have

$$
\text { meas } E_{n}>\frac{\varepsilon_{n}}{q},
$$


where $E_{n}=E\left(r_{n}, a\right) \bigcap\left(\alpha_{j}+\varepsilon_{n}, \alpha_{j+1}-\varepsilon_{n}\right)$. We can assume that (7.2) holds for all $n$. Thus, it follows from the definition of $E(r, a)$ that

$$
\begin{aligned}
& \int_{\alpha_{j}+\varepsilon_{n}}^{\alpha_{j+1}-\varepsilon_{n}} L\left(r_{n}, \theta, a, G\right) d \theta \geq \int_{E_{n}} L\left(r_{n}, \theta, a, G\right) \mathrm{d} \theta \\
&>\operatorname{meas}\left(E_{n}\right) \frac{\delta}{4} T\left(r_{n}, G\right)>\frac{\delta \varepsilon_{n}}{4 q} T\left(r_{n}, G\right) .
\end{aligned}
$$

On the other hand, by Lemma 4.5, we have

$$
\int_{\alpha_{j}+\varepsilon_{n}}^{\alpha_{j+1}-\varepsilon_{n}} L\left(r_{n}, \theta, a, G\right) \mathrm{d} \theta=O\left(r_{n}^{\eta+\varepsilon_{n}}\right) .
$$

Combining (7.3) with (7.4) gives

$$
\frac{\delta \varepsilon_{n}}{4 q} T\left(r_{n}, G\right)<O\left(r_{n}^{\eta+\varepsilon_{n}}\right) .
$$

Thus, $\mu(f) \leq \eta<\infty$, and Theorem 2.3 follows from Theorem 2.2.

Acknowledgements The author is supported by Grants (No.11231009, 11371363, 11501563, 11571049) of NSFC of China.

Open Access This article is distributed under the terms of the Creative Commons Attribution 4.0 International License (http://creativecommons.org/licenses/by/4.0/), which permits unrestricted use, distribution, and reproduction in any medium, provided you give appropriate credit to the original author(s) and the source, provide a link to the Creative Commons license, and indicate if changes were made.

\section{References}

1. Edrei, A., Fuchs, W.H.J.: Bounds for the number of deficient value of certain classes of meromorphic functions. Proc. Lond. Math. Soc. 12, 315-344 (1962)

2. Edrei, A.: Meromorphic functions with three radially distributed values. Trans. Am. Math. Soc. 78, 276-293 (1955)

3. Goldberg, A.A.: Some questions of value distribution theory (in Russian) Appendix to the Russian transl. of H.Wittich, Neuere Untersuchungen über eindeutige analytische Funktionen, Fizmargiz, Moscow (1960)

4. Goldberg, A.A.: Distribution of the value of meromorphic functions with poles attracted to a system of rays. Ukrainskii Math. Zhur. 41(6), 634-638 (1989)

5. Goldberg, A.A., Ostrovskii, I.V.: The distribution of values of meromorphic functions(in Russian), Izdat. Nauk. Moscow, 1970. English translation by Mikhail Ostrovskii, Translations of Mathematical Monographs, 236, American Mathematical Society, Providence, RI (2008)

6. Hayman, W.K.: Meromorphic functions. Oxford (1964)

7. Niino, K.: General defect relations of holomorphic curves. Trans. Am. Math. Soc. 1, 99-113 (1985)

8. Niino, K.: Spread relation and value distribution in an angular domain of holomorphic curves. Kodai Math. Sem. Rep. 28, 361-371 (1977)

9. Petrenko, V.P.: Entire curves (in Russian). Vishcha Shkola, Kharkov (1984)

10. Petrenko, V.P., Hussain, M.: On the growth of entire curves. Izv. Akad. Nauk SSSR Ser. Mat. Tom 37, 467-478 (1973)

11. Petrenko, V.P.: Growth and distribution of values of algebroid functions. Mathmaticheskie Zametki 26(4), 513-522 (1979) 
12. Wu, S.J.: On the argument distribution and growth of meromorphic functions. Sci. China 6, 565-575 (1993)

13. Wu, S.J.: Distribution of the $(0, \infty)$ accumulative lines of meromorphic functions. Chin. Ann. Math. 15B, 453-462 (1994)

14. Yang, C.C., Lin, B.Q.: On the complete meromorphic functions. Kodai Math. J. 15, 341-353 (1992)

15. Zheng, J.H.: On transcendental meromorphic functions with radially distributed values. Sci. China Ser. A. Math. 47(3), 401-416 (2004)

16. Zheng, J.H.: Value distribution of meromorphic functions. Tsinghua University Press, Beijing (2010) 